PROCEEDINGS OF THE

AMERICAN MATHEMATICAL SOCIETY

Volume 139, Number 2, February 2011, Pages 473-484

S 0002-9939(2010)10469-0

Article electronically published on July 8, 2010

\title{
BOUNDARY DIFFERENTIAL RELATIONS FOR HOLOMORPHIC FUNCTIONS ON THE DISC
}

\author{
MIRAN ČERNE AND MATEJ ZAJEC
}

(Communicated by Franc Forstneric)

\begin{abstract}
The existence of solutions of boundary differential relations for holomorphic functions on the disc $\Delta$ is considered. First we prove that for an arbitrary continuous positive function $\Phi$ on the complex plane $\mathbb{C}$ there exists a disc algebra function $f \in A(\Delta)$ such that $\left|f^{\prime}\right|=\Phi(f)$ on $\partial \Delta$. Assuming some smoothness, the existence result is also proved for a quite general differential relation $\rho\left(\xi, f^{\prime}(\xi)\right)=\Phi(\xi, f(\xi)), \xi \in \partial \Delta$, where $\rho$ is a defining function for a family of Jordan curves in $\mathbb{C}$ containing point 0 in its interior and $\Phi$ is a bounded positive function on $\partial \Delta \times \mathbb{C}$.
\end{abstract}

\section{INTRODUCTION}

Let $\Delta$ denote the unit disc in the complex plane $\mathbb{C}$. In this paper we consider the question of existence of a holomorphic function $f \in \mathcal{O}(\Delta)$ on the unit disc with some boundary regularity which over the boundary of the unit disc $\xi \in \partial \Delta$ satisfies a certain nonlinear differential relation

$$
\left|f^{\prime}(\xi)\right|=\Phi(f(\xi)) .
$$

The origin of these type of results is the paper by Beurling [6], in which he considers an extension of the Riemann mapping theorem. For a given bounded positive continuous function $\Phi$ he proved that there is a one-to-one holomorphic solution of problem (1) satisfying additional 'Riemann mapping' condition $f(0)=0$. See also [2, 3, 4, 17, 21].

The beauty of Beurling's result comes from the fact that not only he was able to find a solution of problem (1), which is in fact the easier part of the problem, but he was able to show the existence of a univalent solution. As Example 1.2 below shows, for a general positive continuous function $\Phi$ there is no even locally univalent solution normalized by $f(0)=0$ to problem (1). For that reason we focus only on the existence of solutions of boundary differential relations.

Our first result shows that the boundedness of function $\Phi$ is not necessary for the existence of solutions of differential relation (1).

Received by the editors February 21, 2010 and, in revised form, March 1, 2010.

2010 Mathematics Subject Classification. Primary 30E25, 35 Q15.

Key words and phrases. Boundary value problem, Riemann-Hilbert problem.

The first author was supported in part by grant Analiza in geometrija P1-0291 from the Ministry of Higher Education, Science and Technology of the Republic of Slovenia.

(C)2010 American Mathematical Society Reverts to public domain 28 years from publication 
Theorem 1.1. Let $\Phi$ be a positive continuous function on $\mathbb{C}$ and let $B$ be a finite Blaschke product with no zero at point 0 . Then there exists a disc algebra function $f \in A(\Delta)$ such that $\left|f^{\prime}\right| \in C(\bar{\Delta})$ and

$$
\left\{\begin{array}{l}
f(0)=0, \\
f^{\prime}(z)=B(z) g(z) \text { on } \bar{\Delta}, \text { where } g \in \mathcal{O}(\Delta) \text { and } g(z) \neq 0 \text { on } \bar{\Delta} \backslash\{0\}, \\
\left|f^{\prime}(\xi)\right|=\Phi(f(\xi)) \text { for every } \xi \in \partial \Delta .
\end{array}\right.
$$

In particular, if $B=1$, then $f$ is locally univalent on $\Delta \backslash\{0\}$.

We see that the boundary value problem

$$
\left\{\begin{array}{l}
f(0)=0, \\
\left|f^{\prime}(\xi)\right|=\Phi(f(\xi)) \text { for every } \xi \in \partial \Delta
\end{array}\right.
$$

has infinitely many distinct solutions for every positive continuous function $\Phi$. In fact we may even prescribe on $\Delta \backslash\{0\}$ finitely many critical points of the solutions to (3).

Example 1.2. One can easily see that for a general function $\Phi$ no locally one-toone holomorphic solutions of problem (3) are possible. Namely, let $f, f(0)=0$, be a holomorphic function on $\Delta$, smooth up to the boundary, and whose derivative has no zeros on $\bar{\Delta}$. We consider the holomorphic function

$$
g(z)=\frac{f(z)}{z f^{\prime}(z)}, \quad g(0)=1
$$

on $\Delta$. By the maximum principle there exists a point $\xi \in \partial \Delta$ such that

$$
|g(\xi)| \geq 1
$$

and hence

$$
|f(\xi)| \geq\left|f^{\prime}(\xi)\right| .
$$

Therefore, if $\Phi$ is a function such that $|w|<\Phi(w)$ for every $w \in \mathbb{C}$, no locally one-to-one holomorphic solutions of problem (3) are possible.

Having this example in mind we state another result in which we show that some sublinear growth of function $\Phi$ still allows locally injective solutions of problem (3). See also [17, 22].

Theorem 1.3. Let $\Phi$ be a positive continuous function on $\mathbb{C}$ such that there exist positive constants $0<c<1$ and $M>0$ so that

$$
\Phi(w) \leq c(|w|+M)
$$

for every $w \in \mathbb{C}$ and let $B$ be a finite Blaschke product. Then there exists a disc algebra function $f \in A(\Delta)$ such that $\left|f^{\prime}\right| \in C(\bar{\Delta})$ and

$$
\left\{\begin{array}{l}
f(0)=0, \\
f^{\prime}(z)=B(z) g(z) \text { on } \bar{\Delta}, \text { where } g \in \mathcal{O}(\Delta) \text { and } g(z) \neq 0 \text { on } \bar{\Delta}, \\
\left|f^{\prime}(\xi)\right|=\Phi(f(\xi)) \text { for every } \xi \in \partial \Delta .
\end{array}\right.
$$

In particular, if $B=1$, then $f$ is locally univalent.

These results can also be given a geometric meaning. Namely, for a given holomorphic function $f$, the function $\Phi(f)$ naturally defines a family of circles over $\partial \Delta$ 
in the following way: for each $\xi \in \partial \Delta$ let $\gamma_{\xi}$ be the circle with center at the point 0 and radius $\Phi(f(\xi))$. The fact that $f$ solves (3) actually means that its derivative solves the Riemann-Hilbert problem given by this family of circles,

$$
f^{\prime}(\xi) \in \gamma_{\xi}
$$

We can generalize this setting. We say that $\left\{\gamma_{\xi}\right\}_{\xi \in \partial \Delta}$ is a $C^{2}$ family of Jordan curves $\gamma_{\xi}$ in $\mathbb{C}$ parametrized by the boundary $\xi \in \partial \Delta$ of the disc if there exists a function $\rho \in C^{2}(\partial \Delta \times \mathbb{C})$ such that

$$
\gamma_{\xi}=\{w \in \mathbb{C} ; \rho(\xi, w)=0\}
$$

and $\partial_{\bar{w}} \rho(\xi, w) \neq 0$ for every $\xi \in \partial \Delta$ and $w \in \gamma_{\xi}$. We call $\rho$ a defining function for the family of Jordan curves $\left\{\gamma_{\xi}\right\}_{\xi \in \partial \Delta}$. Recall also that the interior of a Jordan curve $\gamma$ in $\mathbb{C}$ is the bounded component of $\mathbb{C} \backslash \gamma$.

Remark 1.4. According to the definition of a $C^{2}$ family of Jordan curves, $\left\{\gamma_{\xi}\right\}_{\xi \in \partial \Delta}$, each curve $\gamma_{\xi}$ has a $C^{2}$ parametrization and they depend in the $C^{2}$ sense on the parameter $\xi$. The $C^{2}$ smoothness in $w$ variable is needed to assure that the operator $f \mapsto \rho(\xi, f(\xi))$ between certain Hölder spaces is of class $C^{1}$ and that one may use the implicit function theorem. The $C^{2}$ smoothness in the parameter $\xi$ could be relaxed for that matter (see [18, Lemma 6.1]); however at least $C^{1}$ smoothness in $\xi$ is needed for appropriate a priori bounds. See the proof of Theorem 1.5 and the Appendix.

Theorem 1.5. Let $\rho: \partial \Delta \times \mathbb{C} \rightarrow \mathbb{R}$ be a nonnegative $C^{2}$ function such that

1. $\partial_{\bar{w}} \rho(\xi, w)=0$ if and only if $w=0$.

2. For every $c>0$ and every $\xi \in \partial \Delta$ the set $\{w \in \mathbb{C} ; \rho(\xi, w)=c\}$ is a Jordan curve containing the point 0 in its interior.

Also, let $\Phi: \partial \Delta \times \mathbb{C} \rightarrow \mathbb{R}$ be a bounded positive function of class $C^{2}$. Then there exists a function $f \in C^{1}(\bar{\Delta})$, holomorphic on $\Delta$, such that

$$
\left\{\begin{array}{l}
f(0)=0, \\
f^{\prime}(z) \neq 0 \text { on } \bar{\Delta} \\
\rho\left(\xi, f^{\prime}(\xi)\right)=\Phi(\xi, f(\xi)) \text { for every } \xi \in \partial \Delta .
\end{array}\right.
$$

\section{Proofs of Results}

Beurling's argument in [6] is quite geometrical and, as was already mentioned in the beginning of the paper, it is not just a result on the existence of a solution of a certain boundary value problem but a result on the existence of a very special, namely, univalent solution of problem (11). The technique of proving our results, in which we are not asking for univalency of solutions, is more in the spirit of existence theorems for differential equations and it was already used in 17. The basic idea is to 'integrate' the boundary differential relation and then find a fixed point of a certain compact operator.

Proof of Theorem 1.1. Let $A(\Delta)$ be the disc algebra of all continuous functions on $\bar{\Delta}$ which are holomorphic on $\Delta$. For $f \in A(\Delta)$ let $U(f)$ be the continuous function on $\bar{\Delta}$, harmonic on $\Delta$, whose boundary values are equal to $\log \Phi(f)$.

Let $V(f)$ be the harmonic conjugate of $U(f)$ on $\Delta$ such that $V(f)(0)=0$ (that is, $V(f)$ is the Hilbert transform of $U(f))$ and let

$$
H(f)=e^{U(f)+i V(f)} .
$$


Let $H_{2}(\Delta)$ denote the Hardy space of all holomorphic functions on the unit disc with squared integrable boundary functions. Then the operator $H(f): A(\Delta) \rightarrow H_{2}(\Delta)$ is continuous and maps bounded sets into bounded sets, because

$$
\begin{aligned}
\|H(f)-H(g)\|_{2} & \leq\left\|e^{U(f)}-e^{U(g)}\right\|_{2}+\left\|e^{U(g)}\right\|_{\infty}\left\|e^{i V(f)}-e^{i V(g)}\right\|_{2} \\
& \leq\|\Phi(f)-\Phi(g)\|_{2}+\left\|e^{U(g)}\right\|_{\infty}\|V(f)-V(g)\|_{2} \\
& \leq\|\Phi(f)-\Phi(g)\|_{2}+\max _{z \in \bar{\Delta}} \Phi(g(z))\|\log \Phi(f)-\log \Phi(g)\|_{2} \\
& \leq\|\Phi(f)-\Phi(g)\|_{\infty}+\max _{z \in \bar{\Delta}} \Phi(g(z))\|\log \Phi(f)-\log \Phi(g)\|_{\infty},
\end{aligned}
$$

where we have used the inequality $\|V(f)-V(g)\|_{2} \leq\|U(f)-U(g)\|_{2}$; see [12, p. 54].

Let $N$ be a positive integer. We define

$$
F_{N}(f)(z)=\int_{0}^{z} \xi^{N} B(\xi) H(f)(\xi) d \xi
$$

as a mapping from $A(\Delta)$ into itself.

Since $H(f) \in L^{2}(\partial \Delta)$, we have that $F_{N}(f) \in L^{1,2}(\partial \Delta)$ belongs to the Sobolev space of $L^{2}(\partial \Delta)$ functions whose weak derivatives are also in $L^{2}(\partial \Delta)$. Since $L^{1,2}(\partial \Delta)$ compactly embeds into the Hölder space $C^{\alpha}(\partial \Delta)$ for $0<\alpha<\frac{1}{2}$, [26], and since $C^{\alpha}(\partial \Delta)$ for $\alpha>0$ compactly embeds into $C(\partial \Delta)$, we get that $F_{N}$ is a compact operator from $A(\Delta)$ into itself.

If we can prove that $F_{N}$ has a fixed point $f$, then the definition of $F_{N}$ implies that

and hence

$$
f^{\prime}(z)=z^{N} B(z) H(f)(z),
$$

$$
\left|f^{\prime}\right|=\Phi(f)
$$

on $|z|=1$. To prove that for an appropriately chosen $N$ the mapping $F_{N}$ has a fixed point we will use Schauder's fixed point theorem [11. Actually we will show that for $N$ large enough the compact mapping $F_{N}$ maps the closed unit ball $\bar{B}(0 ; 1) \subset A(\Delta)$ into itself. Using parametrization $\xi=z t$ we get

$$
F_{N}(f)(z)=z^{N+1} \int_{0}^{1} t^{N} B(z t) H(z t) d t .
$$

Thus

$$
\left|F_{N}(f)(z)\right| \leq|z|^{N+1} \int_{0}^{1} t^{N}|B(z t)| e^{U(f)(z t)} d t \leq \frac{1}{N+1} \max _{\partial \Delta} \Phi(f)
$$

by the maximum principle for the harmonic function $U(f)$. Therefore

$$
\max _{\bar{\Delta}}\left|F_{N}(f)\right| \leq \frac{1}{N+1} \max _{\partial \Delta} \Phi(f) .
$$

Let

$$
M=\max _{|w| \leq 1} \Phi(w) .
$$

If we choose $N$ so large that $M \leq N+1$, the assumptions of the Schauder's fixed point theorem are fulfilled and the theorem is proved.

Remark 2.1. The proof of Theorem 1.1 shows that even for a fixed finite Blaschke product $B$ with no zero at the point 0 there are actually infinitely many different solutions of problem (3). 
Example 2.2. Let us consider the case where $\Phi$ depends only on $|w|$. Then we can try to find solutions of (3) in the form $f(z)=\lambda z^{N}$ for some positive integer $N$ and constant $\lambda>0$. Hence on $\partial \Delta$ we get the following condition:

$$
\lambda N=\Phi(\lambda) .
$$

For every positive continuous function $\Phi$ there exists $N_{0}$ such that for $N \geq N_{0}$ equation (6) has a solution $\lambda$.

Theorem 1.3 shows that even for some unbounded functions $\Phi$ one can get a locally one-to-one solution of problem (3); however, the growth of $\Phi$ is quite limited.

Proof of Theorem [1.3. We start as in the proof of Theorem 1.1] by defining $U(f)$, $V(f)$ and $H(f)$. Then we define

$$
F(f)(z)=\int_{0}^{z} B(\xi) H(f)(\xi) d \xi,
$$

which is a compact operator from $A(\Delta)$ into itself; see the proof of Theorem 1.1. We are again in search for a fixed point of the operator $F$. This time we study the set of solutions of the equation

$$
f=\lambda F(f)
$$

for some $\lambda \in[0,1]$. If $f \in A(\Delta)$ solves (7), then we have

$$
\left|f^{\prime}\right|=\lambda \Phi(f)
$$

on $\partial \Delta$. The sublinear assumption on $\Phi$ then implies

$$
\left|f^{\prime}(z)\right| \leq \lambda c(|f(z)|+M)
$$

for every $z \in \partial \Delta$. Hence

$$
\max _{\partial \Delta}\left|f^{\prime}\right| \leq \lambda c\left(\max _{\partial \Delta}|f|+M\right)
$$

and so, because

$$
f(z)=\int_{0}^{z} f^{\prime}(\xi) d \xi
$$

we get

$$
\max _{\partial \Delta}|f| \leq \lambda c\left(\max _{\partial \Delta}|f|+M\right) .
$$

Therefore we get the following a priori bound for solutions of equation (7):

$$
\max _{\partial \Delta}|f| \leq \frac{\lambda c M}{1-\lambda c} \leq \frac{c M}{1-c} .
$$

By the Leray-Schauder's principle [27] (or Leray-Schauder's degree [11]) the equation $f=F(f)$ has a solution.

Example 2.3. It is known, [6, that there are examples of bounded positive continuous functions $\Phi$ such that there is more than one solution of problem (44). As an unbounded example one can consider

$$
\Phi(z)=\left\{\begin{array}{l}
(1 / 4)|z|^{2}+(3 / 4) \text { for }|z| \leq 3 \\
(3 / 4)|z|+(3 / 4) \text { for }|z|>3
\end{array}\right.
$$

where $f_{1}(z)=z$ and $f_{2}(z)=3 z$ are solutions of problem (44). However, we do not know of an example of a function $\Phi$ satisfying the assumptions of Theorem 1.3 and such that there is no univalent solution of problem (4). 
The proof of Theorem 1.5 follows similar ideas as the proofs of the previous theorems, but the technique has to be adapted to the case of general Jordan curves $\gamma_{\xi}, \xi \in \partial \Delta$. In addition, the results on Riemann-Hilbert problems we intend to use, [16, 18, 25, 26, work only in the case of some smoothness of the defining function $\rho$.

Before we proceed let us recall some results on the Riemann-Hilbert problems on the disc. Let $0<\alpha<1$. If we denote by $K$ either $\bar{\Delta}$ or $\partial \Delta$, then $C^{\alpha}(K)$ is the algebra of all Hölder continuous functions on $K$ equipped with the norm

$$
\|f\|_{\alpha}=\max _{z \in K}|f(z)|+\sup _{\substack{z, w \in K \\ z \neq w}} \frac{|f(z)-f(w)|}{|z-w|^{\alpha}}<\infty .
$$

Similarly we denote by $C^{1, \alpha}(K)$ the space of all differentiable functions on $K$ whose first derivatives are in $C^{\alpha}(K)$ equipped with the norm

$$
\|f\|_{1, \alpha}=\sum_{|j| \leq 1}\left\|D^{j} f\right\|_{\alpha} .
$$

As before let $A(\Delta)$ be the disc algebra and let $A(\partial \Delta)=\left\{\left.f\right|_{\partial \Delta} ; f \in A(D)\right\}$. For $0<\alpha<1$ we set

$$
A^{\alpha}(\Delta)=C^{\alpha}(\bar{\Delta}) \cap A(\Delta) \text { and } A^{1, \alpha}(\Delta)=C^{1, \alpha}(\bar{\Delta}) \cap A(\Delta) .
$$

Also

$$
A^{\alpha}(\partial \Delta)=C^{\alpha}(\partial \Delta) \cap A(\partial \Delta) \text { and } A^{1, \alpha}(\partial \Delta)=C^{1, \alpha}(\partial \Delta) \cap A(\partial \Delta) .
$$

Recall that we may identify a function $f$ in $A^{\alpha}(\Delta)$ with $\left.f\right|_{\partial \Delta}$ in $A^{\alpha}(\partial \Delta)$ and vice versa; similarly for functions in $A^{1, \alpha}(\partial \Delta)$ and $A^{1, \alpha}(\Delta)$, [19, pp. 363-364]. We also define

$$
A^{1}(\Delta)=C^{1}(\bar{\Delta}) \cap A(\Delta)
$$

as a subspace of $C^{1}(\bar{\Delta})$, the space of continuously differentiable functions on $\bar{\Delta}$ equipped with the norm

$$
\|f\|_{1}=\sum_{|j| \leq 1}\left\|D^{j} f\right\|_{\infty}
$$

Let $\left\{\gamma_{\xi}\right\}_{\xi \in \partial \Delta}$ be a family of $C^{2}$ Jordan curves in $\mathbb{C}$ given by the defining function $\rho \in C^{2}(\partial \Delta \times \mathbb{C})$. If all curves $\gamma_{\xi}$ contain the point 0 in its interior, then [16, 25, 26] there exists a holomorphic function $h \in A^{1, \alpha}(\Delta)$ with no zeros on $\bar{\Delta}$ which solves the corresponding Riemann-Hilbert problem

$$
h(\xi) \in \gamma_{\xi}, \quad \xi \in \partial \Delta .
$$

In terms of the defining function $\rho$ this means that $h$ satisfies the equation

$$
\rho(\xi, h(\xi))=0, \quad \xi \in \partial \Delta .
$$

The set of such solutions of the Riemann-Hilbert problem is $C^{1}$ diffeomorphic to the circle $S^{1}$, and for each point $w_{1} \in \gamma_{1}$ there exists exactly one solution of the Riemann-Hilbert problem with no zeros passing through $w_{1}$; that is, $h(1)=w_{1}$. Moreover, as a consequence of the implicit function theorem, one gets that these solutions depend continuously and even in the $C^{1}$ sense in $A^{\alpha}(\Delta)$ on parameters and defining function $\rho$. See [16, 18, 25, 26, for more details. These results were also used for the description of the polynomial hull of the maximal real torus $T=$ $\bigcup_{\xi \in \partial \Delta}\left(\{\xi\} \times \gamma_{\xi}\right),[16,24,26]$. 
Proof of Theorem 1.5. The assumptions on $\rho$ imply that for every $c>0$ and every $\xi \in \partial \Delta$ the set $\{w \in \mathbb{C} ; \rho(\xi, w)=c\}$ is a Jordan curve in $\mathbb{C}$ containing the point 0 in its interior.

Choose a point $w_{0}$ such that $\rho\left(1, w_{0}\right)=1$. The solution of the gradient flow

$$
\dot{w}=-\nabla \rho(1, w)
$$

which passes through the point $w_{0}$ gives a $C^{1}$ curve in $\mathbb{C}$ that intersects every level set $\rho(1, w)=c, c>0$ exactly once. Reparametrizing this curve we get a $C^{1}$ curve

$$
w_{1}:(0, \infty) \longrightarrow \mathbb{C}
$$

such that

$$
w_{1}(c) \in\{w \in \mathbb{C} ; \rho(1, w)=c\}=: \gamma_{1}^{c} .
$$

For $f \in A^{1}(\Delta)$ consider the Riemann-Hilbert problem

$$
\rho(\xi, w)=\Phi(\xi, f(\xi)), \quad \xi \in \partial \Delta .
$$

Let $H(f)$ be the solution with no zeros on $\bar{\Delta}$ such that $H(f)(1)=w_{1}(\Phi(1, f(1)))$. The existence of such a solution if $f$ were of class $C^{2}$ would be guaranteed by the results from [16, 25, 26. But since the right-hand side of (9) is only a function of class $C^{1}(\partial \Delta)$, we cannot directly apply these results. However, the proof of Forstnerič [16] combined with some results in [10] yields a solution in this case. See Lemma 3.1 and Corollary 3.2 in the Appendix.

Equations (9) define a family of $C^{2}$ Jordan curves $\left\{\gamma_{\xi}\right\}_{\xi \in \partial \Delta}$ in $\mathbb{C}$ which depend in the $C^{1}$ sense on $\xi \in \partial \Delta$. All these curves put together,

$$
T=\bigcup_{\xi \in \partial \Delta}\left(\{\xi\} \times \gamma_{\xi}\right),
$$

form a $C^{1}$ maximal real torus in $\partial \Delta \times \mathbb{C}$. By a result of Čirka, Coupet and Sukhov [10. Cor.1.5] we get that every $A(\Delta)$ solution of the Riemann-Hilbert problem, and even every bounded holomorphic disc in $\mathbb{C}^{2}$ whose cluster set for each $\xi \in \partial \Delta$ lies in $T$, is in $A^{\alpha}(\Delta)$ for every $0<\alpha<1$. So the operator $H$ maps $A^{1}(\Delta)$ into $A^{\alpha}(\Delta)$ for any $0<\alpha<1$.

Let $R>0$ and let $P=\partial \Delta \times \overline{\Delta(0, R)}$. Then there exist positive constants $0<m<M<\infty$ such that

$$
m \leq \Phi(\xi, w) \leq M
$$

for every pair $(\xi, w) \in P$. By Lemma 3.1 in the Appendix (see also 16]) we have that there exists a constant $C>0$ such that for every $f \in A^{1}(\Delta)$ with $\|f\|_{1} \leq R$ we have

$$
\|H(f)\|_{\alpha} \leq C\left(\left\|\frac{\partial}{\partial \theta}\left(\Phi\left(e^{i \theta}, f\left(e^{i \theta}\right)\right)\right)\right\|_{\infty}+1\right) \leq C K\left(\|\Phi\|_{C^{1}(P)}\right)\left(\left\|f^{\prime}\right\|_{\infty}+1\right),
$$

where $K\left(\|\Phi\|_{C^{1}(P)}\right)$ is a constant which depends on the sup norms of the first derivatives of the function $\Phi$ on $P$. Hence $H$ maps bounded subsets of $A^{1}(\Delta)$ into bounded subsets of $A^{\alpha}(\Delta)$.

Let $\psi: \mathbb{C} \rightarrow \mathbb{R}$ be a $C^{1}$ defining function for the curve $t \mapsto w_{1}(t)$. The implicit function theorem for Banach spaces applied to the continuously differentiable mapping [18, 20]

$$
G: A^{\alpha}(\Delta) \times A^{1}(\Delta) \longrightarrow C^{\alpha}(\partial \Delta) \times \mathbb{R}
$$

defined by

$$
G(h, g)(\xi)=(\rho(\xi, h(\xi))-\Phi(\xi, g(\xi)), \psi(h(1)))
$$


at a point $(H(f), f)$ implies the continuity of $H$ as a map from $A^{1}(\Delta)$ into $A^{\alpha}(\Delta)$ for any $0<\alpha<1$. Namely, the partial derivative of $G(h, g)$ at a point $(H(f), f)$ acting on a vector $k$ at $\xi \in \partial \Delta$ is

$$
\left(\left(D_{h} G\right)(H(f), f) k\right)(\xi)=\left(2 \operatorname{Re}\left(\partial_{w} \rho(\xi, H(f)(\xi)) k(\xi)\right), 2 \operatorname{Re}\left(\partial_{w} \psi(H(f)(1)) k(1)\right)\right) .
$$

Recall [16, 25, 26] that since $H(f)$ is a solution of the Riemann-Hilbert problem (91) with no zeros, the winding number of the $C^{\alpha}$ function on $\partial \Delta$,

$$
a: \xi \longmapsto 2\left(\partial_{w} \rho\right)(\xi, H(f)(\xi)),
$$

is 0 , and hence it can be written in the form $a=r e^{p}$, where $r \in C^{\alpha}(\partial \Delta)$ is a positive real function and $p \in A^{\alpha}(\partial \Delta)$. Also, we know that the gradients $2\left(\partial_{\bar{w}} \rho\right)(1, H(f)(1))$ and $2\left(\partial_{\bar{w}} \psi\right)(H(f)(1))$ are perpendicular.

Let $(u, t) \in C^{\alpha}(\partial \Delta) \times \mathbb{R}$. Then the only solution of the equation

$$
\left(D_{h} G\right)(H(f), f) k=(u, t)
$$

is

$$
k=e^{-p}\left(\frac{u}{r}+i V\left(\frac{u}{r}\right)+i C\right),
$$

where the real constant $C$ is determined from the equation

$$
t=2 i\left(\partial_{w} \psi\right)(H(f)(1)) e^{-p(1)}\left(V\left(\frac{u}{r}\right)(1)+C\right) .
$$

Here, $V\left(\frac{u}{r}\right)$ is the Hilbert transform of $\frac{u}{r}$. Also, the product $i\left(\partial_{w} \psi\right)(H(f)(1)) e^{-p(1)}$ is real because the gradients $2\left(\partial_{\bar{w}} \rho\right)(1, H(f)(1))$ and $2\left(\partial_{\bar{w}} \psi\right)(H(f)(1))$ are perpendicular.

Hence $\left(D_{h} G\right)(H(f), f)$ is an isomorphism as a map from $A^{\alpha}(\partial \Delta)$ into $C^{\alpha}(\partial \Delta) \times$ $\mathbb{R}$, and for each $\widetilde{f}$ close to $f$ there exists exactly one solution $H(\widetilde{f})$ of (9) satisfying the condition $H(\widetilde{f})(1)=w_{1}(\Phi(1, \widetilde{f}(1)))$.

Now we define the operator

$$
F(f)(z)=\int_{0}^{z} H(f)(\xi) d \xi,
$$

which maps $A^{1}(\Delta)$ continuously into $A^{1, \alpha}(\Delta)$ and which takes bounded subsets of $A^{1}(\Delta)$ into bounded subsets of $A^{1, \alpha}(\Delta)$. Since the space $A^{1, \alpha}(\Delta)$ compactly embeds into $A^{1}(\Delta)$, we get that $F$ is a compact nonlinear operator from $A^{1}(\Delta)$ into itself.

Finally we show that the image of $F: A^{1}(\Delta) \rightarrow A^{1}(\Delta)$ is bounded. Since $\Phi$ is a bounded function, every solution of the Riemann-Hilbert problem (9) is by the maximum principle uniformly bounded by a constant depending only on $\rho$ and $\Phi$. Therefore $F(f)$ and $F(f)^{\prime}=H(f)$ are bounded by a uniform constant depending only on $\rho$ and $\Phi$, and hence $F$ takes the whole space $A^{1}(\Delta)$ into a finite ball. By the Schauder's fixed point theorem $F$ has a fixed point $f$. Therefore $f^{\prime}=H(f)$ on $\bar{\Delta}$ and hence

on $\partial \Delta$

$$
\rho\left(\xi, f^{\prime}(\xi)\right)=\Phi(\xi, f(\xi))
$$

Remark 2.4. The proof can be adapted to the case of finding solutions of (5) with prescribed finitely many critical points on $\Delta$. Namely, each time we choose a solution of a Riemann-Hilbert problem we can choose a solution $H(f)$ with prescribed zeros 16,25 . 
Remark 2.5. If all fibers $\gamma_{\xi}, \xi \in \partial \Delta$, are convex curves, one knows that the set of points $h(0)$ where $h$ runs over all solutions with no zeros of the Riemann-Hilbert problem is again a convex curve, [1, 16. Hence in the case of circles (Theorem 1.1, Theorem 1.3) we were able to choose uniquely a solution $H(f)$ for which $H(f)(0)$ is positive. But in the case of nonconvex curves $\gamma_{\xi}$ there is no natural way to define the value of $H(f)$ at the point 0 . Thus we decided to choose a solution of the Riemann-Hilbert problem with the prescribed value at $\xi=1$.

Remark 2.6. One can also ask for the existence of solutions of boundary differential relations on an annulus or even on an arbitrary smoothly bounded planar domain. Trying to adapt our proofs to these cases one encounters at least two technical problems. The first is that although the Riemann-Hilbert problems over multiply connected domains are solvable [5, 7, 8, 13, 14, 15, 23], their solutions cannot, in general, be chosen in a continuous way. The second problem seems to be the fact that a holomorphic function on a multiply connected domain does not, in general, have a primitive function. Nevertheless, the question of existence of solutions of boundary differential relations on multiply connected planar domains seems quite intriguing.

\section{Appendix}

To make the paper more self-contained and to extend the discussion on the Riemann-Hilbert problem, we present some a priori estimates needed to prove the existence result for the solutions of the Riemann-Hilbert problem. We very closely follow the proof of Forstnerič in [16, Theorem 5] and only make necessary changes and observations.

Lemma 3.1. Let $\rho \in C^{2}(\partial \Delta \times \mathbb{C})$ be a nonnegative function which satisfies the assumptions of Theorem 1.5. Let $0<m<M<\infty$ be positive constants and let $0<\alpha<1$. Then there exists a constant $C>0$ such that for every $c \in C^{1}(\partial \Delta)$ for which $m \leq c(\xi) \leq M$ for every $\xi \in \partial \Delta$ and every solution $h \in A(\Delta)$ of the Riemann-Hilbert problem

$$
\rho(\xi, h(\xi))=c(\xi), \quad \xi \in \partial \Delta
$$

with no zeros on $\bar{\Delta}$, we have

$$
\|h\|_{\alpha} \leq C\left(\left\|\frac{\partial c}{\partial \theta}\right\|_{\infty}+1\right) .
$$

Proof. Let us first assume that $c \in C^{2}(\partial \Delta)$. For the proof we identify $\rho\left(e^{i \theta}, w\right)$ with $\rho(\theta, w), h\left(e^{i \theta}\right)$ with $h(\theta)$ and $c\left(e^{i \theta}\right)$ with $c(\theta)$. We denote by $\nu(\theta, w)=2 \partial_{\bar{w}} \rho(\theta, w)$ the $w$-gradient of $\rho(\theta, w)$ which is nonvanishing on $\mathbb{C} \backslash\{0\}$.

By a result of Cirka [9] we have $h \in A^{1, \alpha}(\Delta)$ and we may differentiate the equation

We get

$$
\rho(\theta, h(\theta))=c(\theta) .
$$

$$
\rho_{\theta}(\theta, h(\theta))+\operatorname{Re}\left(\frac{\partial h}{\partial \theta}(\theta) \overline{\nu(\theta, h(\theta))}\right)=\frac{\partial c}{\partial \theta}(\theta) .
$$

Since $h$ has no zeros on $\bar{\Delta}$, there exists $g \in A^{1}(\Delta)$ such that $h=e^{g}$.

We introduce the function $\eta(\theta, w)=w \overline{\nu(\theta, w)}$ on $\partial \Delta \times \mathbb{C}$. Hence

$$
\rho_{\theta}(\theta, h(\theta))+\operatorname{Re}\left(\frac{\partial g}{\partial \theta}(\theta) \eta(\theta, h(\theta))\right)=\frac{\partial c}{\partial \theta}(\theta) .
$$


Observe that $\frac{\partial g}{\partial \theta}$ extends as a holomorphic function to $\Delta$ with a zero at the point 0 .

By assumption all Jordan curves $\gamma_{\xi}=\{w \in \mathbb{C} ; \rho(\xi, w)=c(\xi)\}$ contain the point 0 in their interior, and hence the mapping

$$
\eta: \partial \Delta \times(\mathbb{C} \backslash\{0\}) \longrightarrow \mathbb{C} \backslash\{0\}
$$

is null homotopic in $\mathbb{C} \backslash\{0\}$. Therefore we can write it as

$$
\eta(\theta, w)=e^{a(\theta, w)+i b(\theta, w)}
$$

where $a$ and $b$ are $C^{1}$ functions on $\partial \Delta \times(\mathbb{C} \backslash\{0\})$.

Let $\widetilde{b}$ be the harmonic conjugate of $b(\theta, h(\theta))$. From (10) we get

$$
\operatorname{Re}\left(\frac{\partial g}{\partial \theta}(\theta) e^{i(b(\theta, h(\theta))+i \widetilde{b}(\theta))}\right)=e^{-\widetilde{b}(\theta)-a(\theta, h(\theta))}\left(\frac{\partial c}{\partial \theta}(\theta)-\rho_{\theta}(\theta, h(\theta))\right) .
$$

From now on, $C$ will denote a universal constant depending on $\rho, \alpha$ and the bounds $m<M$, and it can change from line to line. Therefore we have

$$
\frac{1}{C} \leq|h(\theta)| \leq C, \quad|b(\theta, h(\theta))| \leq C, \quad\left|e^{-a(\theta, h(\theta))}\right| \leq C, \quad\left|\rho_{\theta}(\theta, h(\theta))\right| \leq C .
$$

These estimates do not give us a uniform bound on $\widetilde{b}$, but nevertheless we can conclude $L^{p}(\partial \Delta)(1 \leq p<\infty)$ boundedness of the function

$$
\left\|e^{ \pm \widetilde{b}(\theta)}\right\|_{p} \leq C
$$

Here one uses [26, Lemma 1.6.15] and Lemmas 7 and 8 from [16].

Let $p \in(2, \infty)$. Using the fact that the Hilbert transform is a bounded operator on the $L^{p}$ space we get from (11) and (12) that

$$
\left\|\frac{\partial g}{\partial \theta}(\theta) e^{i(b(\theta, h(\theta))+i \widetilde{b}(\theta))}\right\|_{p} \leq C\left(\left\|\frac{\partial c}{\partial \theta}\right\|_{\infty}+1\right) .
$$

Observe that

and so

$$
\frac{\partial h}{\partial \theta}=h \frac{\partial g}{\partial \theta}=h e^{-i(b+i \tilde{b})}\left(\frac{\partial g}{\partial \theta} e^{i(b+i \tilde{b})}\right)
$$

$$
\left\|\frac{\partial h}{\partial \theta}\right\|_{\frac{p}{2}} \leq C\left\|e^{\widetilde{b}}\right\|_{p}\left\|\frac{\partial g}{\partial \theta} e^{i(b+i \widetilde{b})}\right\|_{p} \leq C\left(\left\|\frac{\partial c}{\partial \theta}\right\|_{\infty}+1\right) .
$$

We know that $L^{1, \frac{p}{2}}(\partial \Delta)$ embeds into $A^{\alpha}(\Delta)$, where $\alpha=1-\frac{2}{p}$. Therefore

$$
\|h\|_{\alpha} \leq C\left(\left\|\frac{\partial c}{\partial \theta}\right\|_{\infty}+1\right)
$$

Now let $c$ be a $C^{1}$ function on $\partial \Delta$ and let $h \in A(\Delta)$ be a solution of the RiemannHilbert problem

$$
\rho(\xi, h(\xi))=c(\xi), \quad \xi \in \partial \Delta .
$$

By [10, Cor. 1.5] we have $h \in A^{\alpha}(\Delta)$.

The function $c$ can be approximated in the $C^{1}$ norm by smooth functions $\widetilde{c}$. The argument above shows that there exists a finite positive constant $C$ such that the estimate

$$
\|\widetilde{h}\|_{\alpha} \leq C\left(\left\|\frac{\partial \widetilde{c}}{\partial \theta}\right\|_{\infty}+1\right)
$$


holds for all nearby smooth functions $\widetilde{c}$ and solutions $\widetilde{h}$ (with no zeros) of the corresponding Riemann-Hilbert problems. By the implicit function theorem for Banach spaces applied to the mapping

$$
G: A^{\alpha}(\Delta) \times C^{1}(\partial \Delta) \longrightarrow C^{\alpha}(\partial \Delta)
$$

defined by

$$
G(k, d)(\xi)=\rho(\xi, k(\xi))-d(\xi)
$$

at the point $(h, c)$, we can find a sequence of solutions of the Riemann-Hilbert problems for nearby smooth functions $\widetilde{c}$ which in the $A^{\alpha}(\Delta)$ space converges to $h$. Hence the estimate (14) also holds for $h$ and $c$.

Corollary 3.2. Let $f \in A^{1}(\Delta)$. Then the Riemann-Hilbert problem

$$
\rho(\xi, w)=\Phi(\xi, f(\xi)), \quad \xi \in \partial \Delta,
$$

has exactly one solution $H(f) \in A^{\alpha}(\Delta)$ with no zeros on $\bar{\Delta}$ and such that

$$
H(f)(1)=w_{1}(\Phi(1, f(1))) .
$$

Proof. Let $f \in A^{1}(\Delta)$ and let $c(\xi)=\Phi(\xi, f(\xi))$. Let $0<\alpha<\widetilde{\alpha}<1$. The argument above shows that there exists a finite positive constant $\widetilde{C}$ such that the estimate

$$
\|\widetilde{h}\|_{\widetilde{\alpha}} \leq \widetilde{C}\left(\left\|\frac{\partial \widetilde{c}}{\partial \theta}\right\|_{\infty}+1\right)
$$

and the estimate (14) hold for all nearby smooth functions $\widetilde{c}$ and solutions $\widetilde{h}$ with no zeros of the corresponding Riemann-Hilbert problems such that $\widetilde{h}(1)=w_{1}(\widetilde{c}(1))$. Hence all these solutions have a uniformly bounded $A^{\widetilde{\alpha}}(\Delta)$ norm, and they form a relatively compact subset of $A^{\alpha}(\Delta)$. Therefore there exists a subsequence which converges in $A^{\alpha}(\Delta)$ to a solution with no zeros of the Riemann-Hilbert problem $\rho(\xi, h(\xi))=c(\xi)$ such that $H(f)(1)=w_{1}(\Phi(1, f(1)))$. By the implicit function theorem and by the uniqueness in the $C^{2}$ case [16] we get that this solution is unique.

\section{REFERENCES}

[1] H. Alexander and J. Wermer, Polynomial hulls with convex fibers, Math. Ann. 271 (1985), 99-109. MR779607 (86i:32025)

[2] F. G. Avkhadiev, Metrics with variable density and inverse boundary value problems (Russian), Trudy Sem. Kraev. Zadacham 25 (1990), 3-23. MR.1084789 (91k:30091)

[3] F. G. Avkhadiev and L. I. Shokleva, Generalizations of a theorem of Beurling and their applications to inverse boundary value problems, Russ. Math. (Iz. VUZ) 38 (1994), 78-81. MR.1301697 (95i:30008)

[4] F. Bauer, D. Kraus, O. Roth and E. Wegert, Beurling's free boundary value problem in conformal geometry, to appear in Israel Jour. Math.

[5] H. Begehr and M. A. Efendiev, On the asymptotics of meromorphic solutions for nonlinear Riemann-Hilbert problems, Math. Proc. Cambridge Philos. Soc. 127 (1999), 159-172. MR:1692479 (2000c:30076)

[6] A. Beurling, An extension of the Riemann mapping theorem, Acta Math. 90 (1953), 117-130. MR0060027 (15:614e)

[7] M. Cerne, Nonlinear Riemann-Hilbert problem for bordered Riemann surfaces, Amer. J. Math. 126 (2004), 65-85. MR.2033564 (2004k:30092)

[8] M. Černe and M. Flores, Generalized Ahlfors functions, Trans. Amer. Math. Soc. 359 (2007), 671-686. MR2255192(2007h:30042)

[9] E. M. Čirka, Regularity of boundaries of analytic sets, Math. Sb. (NS) 117(159) (1982), 291-336; English translation: Math. USSR Sb. 45 (1983), 291-335. MR648411 (83f:32009) 
[10] E. M. Čirka, B. Coupet and A. B. Sukhov, On boundary regularity of analytic discs, Michigan Math. J. 46 (1999), 271-279. MR.1704142 (2000f:32022)

[11] K. Deimling, Nonlinear functional analysis, Springer-Verlag, Berlin, 1985. MR787404 $(86 \mathrm{j}: 47001)$

[12] P. L. Duren, Theory of $H^{p}$ spaces, Academic Press, New York and London, 1970. MR 0268655 $(42: 3552)$

[13] M. A. Efendiev and W. L. Wendland, Nonlinear Riemann-Hilbert problems for multiply connected domains, Nonlinear Anal. 27 (1996), 37-58. MR1390711(97h:30057)

[14] M. A. Efendiev and W. L. Wendland, Nonlinear Riemann-Hilbert problems without transversality, Math. Nachr. 183 (1997), 73-89. MR1434976 (98b:30038)

[15] M. A. Efendiev and W. L. Wendland, Nonlinear Riemann-Hilbert problems for doubly connected domains and closed boundary data, Topol. Methods Nonlinear Anal. 17 (2001), 111124. MR:1846981 (2002d:30045)

[16] F. Forstnerič, Polynomial hulls of sets fibered over the circle, Indiana Univ. Math. J. 37 (1988), 869-889. MR982834 (90g:32018)

[17] R. Fournier and S. Ruscheweyh, Free boundary value problems for analytic functions in the closed unit disk, Proc. Amer. Math. Soc. 127 (1999), 3287-3294. MR1618666 (2000b:30059)

[18] J. Globevnik, Perturbation by analytic discs along maximal real submanifolds of $\mathbb{C}^{N}$, Math. Z. 217 (1994), 287-316. MR1296398 (95j:32031)

[19] G. M. Goluzin, Geometric theory of functions of a complex variable, Trans. of Math. Monographs, Vol. 26, Amer. Math. Soc., Providence, RI, 1969. MR0247039 (40:308)

[20] C. D. Hill and G. Taiani, Families of analytic discs in $\mathbb{C}^{n}$ with boundaries on prescribed CR submanifold, Ann. Scuola. Norm. Sup. Pisa 5 (1978), 327-380. MR501906 (80c:32023)

[21] M. J. Huntey, N. J. Moh and D. E. Tepper, Uniqueness theorems for some free boundary problems in univalent functions, Complex Var. Theory Appl. 48 (2003), 607-614. MR1988687 (2004k:35421)

[22] R. Kühnau, Längentreue Randverzerrung bei analytischer Abbildung in hyperbolischer und Sphärischer Metrik, Mitt. Math. Sem. Giessen 229 (1997), 45-53. MR1439207 (98g:30011)

[23] F. G. Maksudov and M. A. Efendiev, The nonlinear Hilbert problem for a doubly connected domain (Russian), Dokl. Akad. Nauk SSSR 290 (1986), 789-791. MR863355 (88j:30088)

[24] Z. Slodkowski, Polynomial hulls in $\mathbb{C}^{2}$ and quasicircles, Ann. Scuola Norm. Sup. Pisa 16 (1989), 367-391. MR1050332 (91m:32016)

[25] A. I. Snirelman, The degree of a quasiruled mapping and a nonlinear Hilbert problem. Mat. Sb. 18 (1972), 373-396; English transl., Math USSR Sb. 18 (1973), 373-396. MR0326521 $(48: 4865)$

[26] E. Wegert, Nonlinear boundary value problems for holomorphic functions and singular integral equations, Mathematical Research 65, Akademie-Verlag, Berlin, 1992. MR.1206907 (94b:30049)

[27] E. Zeidler, Applied functional analysis (Applications to mathematical physics), Applied Mathematical Sciences 108, Springer, Berlin, 1995. MR.1347691(96i:00005)

Department of Mathematics, University of Ljubljana, Jadranska 21, 1111 Ljubljana, SLOVENIA

E-mail address: miran.cerne@fmf.uni-lj.si

Institute of Mathematics, Physics and Mechanics, Jadranska 19, 1111 Luubluana, SLOVENIA

E-mail address: matej.zajec@imfm.uni-lj.si 